\title{
$\underline{\text { Relating pessimistic memory predictions to Alzheimer's disease brain structure }}$
}

Sarah Genon ${ }^{1,2}$, Jessica Simon ${ }^{1}$, Mohamed Ali Bahri ${ }^{1}$, Fabienne Collette ${ }^{1,6}$, Céline Souchay ${ }^{5}$, Mathieu Jaspar ${ }^{1}$, Christine Bastin ${ }^{1 *}$ and Eric Salmon ${ }^{1,4^{*}}$.

${ }^{1}$ GIGA-CRC in vivo imaging, University of Liège, Belgium

${ }^{2}$ Institute of Neuroscience und Medicine (INM-1), Research Centre Juelich, Juelich, Germany

${ }^{4}$ Memory Centre, Service of Neurology, CHU Liège, Belgium

${ }^{5}$ Laboratoire de Psychologie et Neurocognition CNRS UMR 5105, Université Pierre MendèsFrance, Grenoble, France

${ }^{6}$ Psychology and Cognitive Neuroscience research unit, University of Liège, Belgium

* Equally contributed

Running title: Metacognitive predictions in Alzheimer's disease

Key words: metacognition; ventrolateral prefrontal cortex; PLS; VBM; monitoring

Number of figures: 5

Number of tables: 1

No supplemental material

\section{Corresponding author:}

Sarah Genon, GIGA-CRC in vivo imaging, University of Liège, Quartier Agora, Allée du 6 Août, B30, 4000 Liège, Belgium, Telephone: 3243665475, Fax: 3243662946, Email: sarah.genon@ulg.ac.be. 


\begin{abstract}
Patients with Alzheimer's disease (AD) show impairment of episodic memory and related metacognitive processes. The present study examined subjective metacognitive judgments preceding objective memory retrieval and investigated the neural correlates of pessimistic predictions for successfully retrieved memories in $\mathrm{AD}$ patients.

$\mathrm{AD}$ patients and healthy older participants provided predictive judgements on their recognition performance before retrieval of famous (semantic) and recently learned (episodic) names. Correlations between grey matter volume (GMV) in T1 images and behavioural scores were examined with multivariate (PLS) and univariate (GLM) analyses in AD patients. AD patients showed a significant proportion of successful name recognition preceded by pessimistic prediction (Prediction_low_hits) in episodic memory. PLS revealed that behavioural pattern in $\mathrm{AD}$ patients was related with a mainly right lateralized pattern of GMV decrease including medial temporal lobe and posterior cingulate cortex, but also right ventrolateral prefrontal cortex (VLPFC). GLM further confirmed that pessimistic prediction negatively correlated with GMV in VLPFC.

Thus, impaired monitoring processes (possibly influenced by inaccurate beliefs) allowing inferences about one's own memory performance are primarily related to decrease GMV in VLPFC in AD patients.
\end{abstract}




\section{I. $\quad$ Introduction}

Patients with Alzheimer's disease (AD) are characterized by severe (although variable) memory impairments. One striking feature highlighted by previous fine examination of memory retrieval of recent memories in $\mathrm{AD}$ patients is the dissociation between automatic and controlled processes with the former being relatively preserved (based on familiarity or fluency for the memory trace) while controlled and conscious access to recently encountered information in memory (i.e. episodic memory retrieval) is particularly impaired (Dalla Barba, 1997; Genon et al., 2014; Genon et al., 2013). One relevant aspect that can be examined to better understand the neurocognitive mechanisms of episodic memory retrieval in AD is the insight or subjective judgment that the patients have on their current memory functioning when they are in the context of retrieving information from memory. The extent to which AD patients have conscious access to the memory trace and are able to use this access to monitor their memory functioning during a memory task can be assessed by the patients' overt metacognitive judgments on their memory performance (Souchay and Moulin, 2009). Traditionally, metamemory theoretical framework (Nelson and Narens, 1990) distinguishes metacognitive judgments that are performed during retrieval attempt, with feeling-of-knowing (FOK), from metacognitive judgments that are performed directly after target retrieval, expressed as judgments of confidence (JOC). The few studies that have examined postretrieval metacognitive judgments (JOC) in AD patients suggested that such metacognitive processes can be relatively preserved (Gallo et al., 2012; Moulin et al., 2003), although it might depend on task-demands and the stage of dementia (Dodson et al., 2011). In contrast, predictive metacognitive judgments (FOK) have been found to be altered in AD-like pathology with AD patients and MCI patients tending to overestimate their abilities (Souchay et al., 2002; Perrotin et al., 2007). Such alteration seems to be specific to judgments related to 
newly formed episodic memories (word pairs; (Souchay et al., 2002)) as studies examining these judgments for general knowledge (semantic memory) suggest that they may be relatively preserved (Backman and Lipinska, 1993; Lipinska and Backman, 1996). Thus, AD patients' abilities to consciously access and monitor the memory content related to recently encountered information are impaired in such a way that predictive judgments on their retrieval performance for these memories are inaccurate.

At the brain level, task-based fMRI studies in healthy young participants showed that predictive judgments on episodic memory retrieval performance engage the medial temporal lobe and posterior medial structures, as well as prefrontal regions supporting monitoring processes (e.g. Maril et al., 2003). In line with those findings, patients with frontal damage showed impaired abilities to predict their episodic memory performance in a FOK paradigm (Pannu and Kaszniak, 2005; Souchay et al., 2006). Therefore, we assume that cerebral perturbations in prefrontal regions may be related to impaired metacognitive processes supporting subjective judgments on the memory content for newly presented information in AD patients. This hypothesis, and more precisely the neural correlates of inaccurate predictive subjective judgments on available memory content reflecting metacognitive dysfunction, can be examined by relating interindividual variations of fine cognitive performance in a prediction-recognition task to cerebral grey matter variations (Kanai and Rees, 2011).

Importantly, a prediction-recognition task should be distinguished from the traditional paradigm used to examine metacognitive judgments at memory retrieval stage, that is, the FOK paradigm. In this latter, the participants are first asked to overtly recall the target associated to a cue and only when they fail, they are asked to indicate whether their subjective feeling of knowing (FOK) the target that they are currently not able to recall. Comparing the feeling of knowing (FOKyes vs FOKno) of the participant to his/her actual performance 
(success-failure) in such paradigm allows measuring the global accuracy of online metacognitive processes in clinical populations (such as AD patients) when compared to healthy controls. However, a fine examination of different combinations of judgment and subsequent memory success/failure may be required to tackle $\mathrm{AD}$ patients' specific impairment and then examine the neural correlates of such alteration. Furthermore, the identification of the neural correlates of metacognitive abilities within a memory task in $A D$ patients is further complicated by the close relationship between memory and metamemory (Nelson and Narens, 1990). Specifically targeting metacognitive alterations within an associative memory task in $\mathrm{AD}$ patients requires performance well above chance level in those patients. In this end, encoding should be deep and successful. That is, efficient encoding should promote persistence of the target memory trace in the subsequent predictionrecognition stage.

In the present study, we designed a new task targeting daily life difficulties of AD such as naming people from their faces and we examined the subjective prediction judgment that preceded correct name recognition. An encoding procedure based on immediate recall and relational encoding of the face and the name ensured deep and efficient encoding of the item. Furthermore, in order to ensure that the revealed prediction-recognition patterns were specific to newly formed memories (i.e. episodic memories), we included a similar task based on famous people (i.e. semantic task) carefully designed to match the episodic task. In the prediction phase, we provided a range of four options to the participants for them to indicate their subjective feeling (Schnyer et al., 2005). This behavioural paradigm allowed examining subjective feeling of failure despite objective memory trace persistence. The corresponding structural correlates at the brain level were then investigated by capitalizing on two statistical approaches and conservative thresholds. Finally, in order to explore whether the revealed behaviour-brain relationship pattern was specific to AD patients, we performed similar Genon et al. 


\section{cognitive-morphologic correlation in a healthy older sample from an independent fMRI}

study.

\section{$\underline{\text { II. Methods and Materials }}$}

\subsection{Participants}

Seventeen healthy older adults $(\mathrm{HO})$ and 23 patients diagnosed with clinical mild probable AD (McKhann et al., 1984) participated in this study. Patients were recruited via Memory Clinics and gave their informed consent to participate to the study. The diagnosis was based on general examination, neurological and neuropsychological assessments and structural neuroimaging. The initial diagnostic neuropsychological assessment covers the main cognitive functions: declarative long-term memory (assessed with two verbal and one visual learning tasks), verbal and spatial working memory (assessed respectively with digit span tasks and a block-taping test), language functions (assessed with a picture naming and fluency tasks), and attention and executive functions (assessed with attention, flexibility and inhibition tasks). A characteristic pattern of cerebral hypometabolism (individual visual analysis of FDG-PET) was used as biomarker (McKhann et al., 2011). HO had no history of neuropsychiatric problems or memory difficulty. Gender was similarly distributed amongst HO (males: $41 \%$ ) and probable $\mathrm{AD}$ groups (males: $43 \% ; \chi^{2}=.02 ; \mathrm{p}=.88$ ). Groups were matched with regard to years spent in formal education [AD: $12.1 \pm 3.7 ; \mathrm{HO}: 12.4 \pm 3.3 ; \mathrm{t}(37)$ $=-0.2 ; p=.85]$ and age [AD: $78.2 \pm 5.6 ; \mathrm{HO}: 76.7 \pm 58.0 ; \mathrm{t}(38)=0.9 ; \mathrm{p}=.39]$. The patients and HO were assessed with the dementia rating scale (Mattis, 1973) and the performance was significantly lower in probable AD patients $(126.7 \pm 6.9)$ than in $\mathrm{HO}(137.1 \pm 4.2 ; \mathrm{T}(36)=-$ 5.4; $\mathrm{p}<.00001)$. The data of one AD patient have not been included in the analyses since her episodic and semantic memory performance matched chance level.

Genon et al. 


\subsection{Memory task}

At the behavioral level, we capitalized on a memory task allowing finely assessing subjective predictions for recently encountered information in AD patients. We designed a memory task intended as ecological in that sense that it aims to target names retrieval as a common daily life memory challenge. This was done by using a retrieval task of the accurate name when a face is presented (Eakin et al., 2014). In order to finely assess primary metacognitive judgments accompanying memory retrieval, the retrieval phase of each item was preceded by a subjective prediction/judgment in which the participant had to choose among four levels to indicate his/her primary subjective judgment. Finally, in order to examine whether judgments pattern for recently encountered information differ from judgments pattern for general knowledge, we used an unique design of face-name memory task but comprising items made of recently encountered unfamous people (episodic items) and items made of famous people (semantic items).

\subsubsection{Materials}

Eighty-five full face photographs of unknown people's face and 85 full face photographs of famous people's face were selected. The background of all the photographs was standardized in a soft grey colour. Additionally, we selected 170 French first and last names from webpages on which the most popular first and last name per decade are registered (http://statbel.fgov.be and http://prenoms.doctissimo.fr/top-prenoms-annee.html). The famous characters were selected based on pre-tests in 30 healthy older people (17 males; $76 \pm 6$ years old; $13 \pm 3$ years of education) in order to avoid ceiling and floor effect in famous faces recognition and naming. They included politicians, pop stars, actors/actresses, and other public figures, either dead or still alive. The unknown characters were selected in order to match the famous characters with regard to age category $\left(\chi^{2}=.37 ; \mathrm{p}=1.00\right)$ and gender $\left(\chi^{2}=\right.$ $.00 ; \mathrm{p}=1.00$ ). Eighty famous characters (face and full name) were used for the semantic 
prediction-recognition test and 80 unknown faces, first and last name were used for the episodic prediction-recognition test. Five famous and unknown characters were used for practice trials. Eighty-five famous full names were selected as lures for the semantic recognition test. The lures were matched to the targets regarding age category, gender and the domain of fame (target distractor). Phonological distractors of the target and the lures were built by changing the consonants of the respective names. Similarly, eighty-five common full names were selected as lures for the episodic recognition test (the lures were matched to the target regarding age category and gender) and eighty-five phonological distractors of the target and lures were built by changing the consonants of the respective names. Of note, the phonological manipulation could not be applied to the people's first names as it would have resulted in uncommon first names in a French speaking community (e.g. changing the consonants of "David" yields "Racif"). All stimuli were presented on a computer screen by using Cogent software running on MATLAB 6.1. (Mathworks Inc., Sherborn, MA).

\subsubsection{Procedure}

The task procedure included a familiarisation phase with the unknown faces, an encoding phase of the episodic materials, a prediction and recognition phase on episodic materials (episodic prediction-recognition test) and a prediction and recognition phase on semantic materials (semantic prediction-recognition test). This procedure is illustrated in Figure 1.

[Figure 1 about here]

Familiarisation phase for faces:

During the familiarisation phase, participants saw the 85 unknown faces. For each face, they were asked to indicate, without time limit, the gender of the person by pressing either the left (male) or the right (female) button on a keyboard.

Genon et al. 
Encoding phase for name-face association:

During the encoding phase, the 85 unknown faces associated with full names were presented to the participant as people for whom they had to learn the full name. For each person, the face and the full name were displayed on the left and the right side of the screen respectively. The participants had first to read aloud the full name of the person, then to say aloud if the name of the person suits his/her face and to explain why (self-paced). Then, for each trial, there was an immediate recall of the full name in which the face was presented and the participant had to recall orally the full name of the person. For each person, when the participants failed at immediate recall, the procedure was repeated for this item until the participants were able to correctly recall the full name. The order of stimuli presentation was counterbalanced across the participants. The participants were told that the encoding phase will be followed by a recognition test.

Prediction-recognition phase with face presentation:

After a 10-minute relaxing break, the instructions and the practice trials followed by the episodic prediction-recognition test were administered. For each face, the participants first saw the face and were asked to indicate to which extent they thought they could recognize the correct full name. The question appeared above the face: "Could you recognize his/her full name?". The participants had to indicate the probability of recognition of the correct name on a four-point scale displayed beside the face: 1* "Not at all" (Null_feeling), 2* "Low probability" (Prediction_low), 3* "High probability" (Prediction_high), 4* "Yes, I know it"(Subjective_recall). The participants were instructed to answer * "Not at all" (Null_feeling) if they felt that they did not know the person at all, as if they had never seen him/her before (which may correspond to an absence of familiarity and of retrieval capacity for the item), * "Low probability" (Prediction_low) if they felt they had seen the face before but they thought that the probability to recognize the correct full name among four possible names was weak, * 
"High probability" (Prediction_high) if they felt very confident about the correct recognition of the name, and * "Yes, I know it" (Subjective_recall) if they have recollected the full name of the person and were able to recall it without any suggestions. These four subjective judgments propositions ensured to account for $*$ Null_feeling: subjective failure to recognize the cue, * Prediction_low: feeling of not being able to recognize the target (i.e., negative prediction), * Prediction_high: prediction of target recognition success despite not feeling currently able to recall the target name, and * Subjective_recall: recall of the target. The participants had to indicate their subjective judgments within an interval of 12 seconds. For each face, once the participants had indicated their subjective judgment, the face was displayed with four propositions of full name: the target (e.g.: "Suzanne Firmin"), the lure (e.g.: "Véronique Pignon"), the phonological distractor of the target (e.g.: "Suzanne Quirtin") and the phonological distractor of the lure (e.g.: "Véronique Biquon"). The participants had to indicate the correct full name within an interval of 9 seconds. The prediction-recognition procedure was exactly the same for the semantic prediction-recognition test (e.g.: target, "Michèle Laroque"; lure, "Valérie Lemercier"; phonological distractor of the target, "Michèle Balof"; phonological distractor of the lure, "Valérie Benépié"). The administration of the tasks was counterbalanced across participants: in half of the participants, the familiarisation phase, encoding phase and the episodic prediction-recognition test were administered first and in the other half of participants, the semantic prediction-recognition test was administered first.

\subsubsection{Behavioral measures}

Memory performance:

Episodic and semantic memory performance was measured by the percentage of correct recognition (i.e. Hits, number of correct recognition/total number of trials) for episodic items

Genon et al. 
and semantic items, respectively. Chance level was $25 \%$ of hits (4-alternative forced-choice paradigm).

Subjective judgments:

We first examined the mean proportions of each judgment (Null_feeling, Prediction_low, Prediction_high, Subjective_recall) for episodic and semantic items separately in each group. In order to investigate the pattern of subjective judgment that preceded accurate memory retrieval, we then examined the proportion of correct recognition that was preceded by each kind of judgement: Null_feeling_hits, Prediction_low_hits, Prediction_high_hits and Subjective_recall_hits. Those indexes were obtained by dividing the number of each kind judgment (followed by correct recognition) by the total number of correct recognition. We thus obtained indexes of accurate and inaccurate primary subjective judgments for subsequently successful recognition expressed as a percentage of the total number of correct recognition. In the current paradigm framework, Null_feeling_hits reflects correct memory retrieval preceded by absolute feeling of not knowing the item (i.e. the cue and therefore the whole face-name item), this latter being likely followed by by-chance target endorsement. In contrast, Prediction_low_hits reflects correct memory retrieval preceded by subjective judgment of quite poor knowledge that will likely result in recognition failure. We were particularly interested in the proportion of correct recognition that was preceded by Prediction_low, i.e. Prediction_low_hits, and the proportion of correct recognition that was preceded by Prediction_high, i,e., Prediction_high_hits. Prediction_low_hits reflect correct memory retrieval preceded by judgement of not knowing the target name (despite knowing the face) or pessimistic prediction of failure. In turn, Prediction_high_hits reflect correct retrieval preceded by judgment of knowing the target name (despite being unable to freely recall it) or accurate prediction of success. As described supra, our cognitive paradigm intended to promote memory performance above chance level in our participants. 
Nevertheless, due to the high number of items to learn ( 85 items), we only expect a marginal

number of subjective recall (and thus Subjective_recall_hits). In a related issue, we could have been interested in predictions preceding false recognitions. However, our materials, in particular semantic materials (i.e. famous characters), had been designed to ensure decent memory performance (but name recall failure) in older people. Therefore, we did not expect a substantial proportion of false recognitions in our participants (in particular for semantic items) that would have allowed us to deeply examine the different predictions for episodic and semantic false retrieval and their interaction with experimental groups.

\subsection{MRI data acquisition and preprocessing}

MRI was performed within one month of the cognitive assessment in $19 \mathrm{AD}$ patients (the MRI could not be performed in three AD patients due to technical problems) with a 3T headonly scanner (Magnetom Allegra, Siemens Medical Solutions, Erlangen, Germany). The patients were equipped with earplugs and their heads were stabilized with foam pads to minimize head motion while a high-resolution T1-weighted anatomical image was acquired with a standard transmit-receive quadrature head coil and following acquisition parameters: TR $7.92 \mathrm{~ms}$, TE $2.4 \mathrm{~ms}$, FA $15^{\circ}, 176$ sagittal slices, FOV $=256 \times 224 \mathrm{~mm}$, voxel size $=1$ mm x 1mm x 1mm (Deichmann et al., 2004).

T1 images were processed using the VBM8 toolbox (http://dbm.neuro.uni-jena.de/vbm.html) implemented in SPM8 (http://www.fil.ion.ucl.ac.uk/spm) using standard settings. Images were corrected for bias-field inhomogenities, segmented into gray matter (GM), white matter (WM) and cerebrospinal fluid (CSF) and adjusted for partial volume effect (with a simplified mixed model of at most two tissue types: GM-WM and GM-CSF). This results in an estimation of the amount (or fraction) of each pure tissue type present in every voxel. Normalization was performed to the Montreal Neurological Institute (MNI) stereotactic space and included non-linear modulation of the segmented images in which the value of each voxel 
is modulated by the Jacobian of the warp field (i.e. Jacobian determinants from the spatial normalization; (Good et al. 2001)).

Finally, the obtained GM images were smoothed with an $8 \mathrm{~mm}$ FWHM Gaussian kernel.

Cognitive-morphologic correlations: Multivariate and univariate analyses

Of note, the cognitive-morphologic correlation analysis could only be performed in $A D$ patients as brain structural data were not acquired in HO. To robustly identify the neural correlates of memory success preceded by weak and strong prediction judgments in our sample of AD patients, we used both a univariate (SPM general linear model, GLM) and a multivariate statistical design (PLS). This latter design is assumed to overcome some limitations of mass-univariate approaches by increasing the sensitivity to detect subtle or spatially distributed effects in brain signals (McIntosh and Lobaugh, 2004). Furthermore, in contrast to the GLM, PLS allows modeling effects of strongly collinear variables (Wegelin, 2000; Ziegler et al., 2013) as may be the proportions of different memory judgments and memory performance in the present study (see result section for further developments). As a matter of fact, our variables of interest, Prediction_low_hits and Prediction_high_hits are collinear as both are proportions of total hits. For example, if a participant has $50 \%$ of Prediction_low_hits, the proportion of Prediction_high_hits cannot be greater than $50 \%$. In other words, Prediction_low_hits and Prediction_high_hits covary to some extent. Therefore, in the current study, in a first explorative step, we used a multivariate analysis. That is, we tested a whole model with PLS. Of note, we included total hits for episodic items in this model to ensure that the significant pattern(s), or latent variable(s), was/were not mainly driven by global episodic memory abilities. To confirm and better understand the relationship between GMV and behavioral variables of interest (Prediction_low_hits and Prediction_high_hits), we then introduced these variables separately in a univariate SPM design. 
In sum, in order to investigate the neural correlates of the prediction-recognition association pattern in AD patients, we used both a multivariate and a univariate statistical design. We first statistically explored how the pattern of GMV was related to our behavioural measures by using regular behavioural PLS and then performed confirmatory univariate analyses to examine in which brain region GMV significantly correlates with each of the selected behavioural measure. Importantly, both analyses were performed when adjusting for the confounding effect of age and dementia ratings (as reflected by total score at the Mattis Dementia Rating Scale) on grey matter values and behavioural measures.

\subsubsection{Multivariate analyses: Regular behavioural PLS analysis}

We performed a regular behavioural PLS analysis using the PLSGUI software (http://www.rotman-baycrest.on.ca/pls/). In line with a previous study examining the relationship between behavioural variables and GMV with a multivariate model (Ziegler et al., 2013), we first adjusted the cognitive variables (Prediction_low_hits, Prediction_high_hits and Hits) and the brain data (as reflected by the 8mmm smoothed GM images) for linear age and dementia ratings by using multiple linear regression. The adjusted cognitive scores Prediction_low_hits, Prediction_high_hits and Hits were then introduced as variables in relation to adjusted GMV. The significance of the latent variables representing covariance of brain and behavioural patterns was assessed with a permutation test (600 permutations) while 500 bootstrap resamples were used to assess the reliability of voxels contributing to the latent variable. In line with previous studies (Walsh et al., 2014), the most reliable voxels contributing to the pattern were examined with a bootstrap ratio of \pm 3.3 (corresponding to $\mathrm{P}$ $<.001)$ and an extent threshold of 250 voxels.

\subsubsection{Univariate analyses: SPM General Linear Model (GLM)}

We performed a multiple regression design assessing correlation between GMV and 1) Genon et al. 
Prediction_low_hits or 2) Prediction_high_hits. Importantly, the regression model included age and dementia ratings as reflected by the total score at the Mattis Dementia Rating Scale as covariates. The significant clusters were then assessed by using Threshold free cluster enhancements (TFCE; Smith and Nichols, 2009) correcting for multiple comparisons using the familywise error (FWE) correction at $P \leq 0.05$ and 5000 permutations.

\subsection{Additional cognitive-morphologic correlations in an independent healthy population}

In order to further explore whether the behavioral measures of interest correlated with GMV in healthy older people, we performed cognitive-morphologic correlation in a sample of 36 healthy older people (HOfmri). Importantly, this sample was similar to the AD and HO samples regarding age (HOfmri: $76.81 \pm 89, F=.8 ; P=.46)$, years spent in formal education (HOfmri: $12.69 \pm .57, \mathrm{~F}=.13 ; P=.88$ ) and gender (HOfmri: $\mathrm{M} / \mathrm{F}=$ 19/17; $\left.\mathrm{X}^{2}=.17 ; P=.92\right)$. Furthermore HO and HOfmri samples had similar scores at the Mattis Dementia Ratings Scale $(138.6 \pm .88, t=-0.9 ; P=0.6)$

This set of data was part of a follow-up functional MRI study examining brain activations related to metacognitive judgments for episodic and semantic memories in healthy aging. The fMRI study was performed with the procedure and material described supra. T1 structural images were acquired and preprocessed as described supra. The GLM analysis used to examine significant correlations between grey matter volume and Prediction_low_hits/Prediction_high_hits was performed with the same parameters than the one used in the AD sample analysis (in particular, age and total score at the Mattis Dementia Rating Scale were introduced as covariates in the GLM design).

The only minor behavioural differences between the two studies are related to the constraints of task-based fMRI: 1) In the fMRI study, the behavioral task was administrated in an fMRI scanner 2) The number of each domain items was slightly 
reduced in the fMRI study (70 episodic items and 70 semantic items) and they were pseudo randomly mixed within a single task with a maximum of three subsequent items for each domain. 3) A random interval of items between prediction and recognition items of each character was used to prevent collinearity in fMRI signal between prediction events and recognition events in the fMRI study.

\section{$\underline{\text { III. Results }}$}

\section{$\underline{\text { 3.1. Memory performance }}$}

Of note, in many cases, the participants were not able to manage the four keyboard keys corresponding to the four judgments/recognition alternatives within the time limit without the help of the experimenter to press the key. That is, in many cases, the participants indicated orally his/her answer to the experimenter who, then, pressed the appropriate keys. Therefore, response time did not reflect participant's abilities and were not used in behavioural and cognitive-morphologic analyses.

Percentages of hits for episodic and semantic tasks are illustrated in Figure 2. A 2(Group) x 2(Task) ANOVA revealed no main effect of the group $[\mathrm{F}(1,37)=2.38 ; P=.13]$, a significant main effect of the task $[\mathrm{F}(1,37)=39.53 ; P=<.01]$ and a significant interaction between the memory task and the group $[\mathrm{F}(1,37=6.95 ; P<.05]$. Post-hoc comparisons (Tukey tests) showed that in $\mathrm{AD}$ patients, episodic memory performance was significantly lower than semantic memory performance $(P<.01)$ and that episodic memory performance of AD patients was significantly lower than episodic memory performance of $\mathrm{HO}(P<.05)$.

[Figure 2 about here]

Genon et al. 
In sum, while $\mathrm{AD}$ patients had significantly lower recognition performance than $\mathrm{HO}$ for episodic items, they reached similar recognition performance for semantic items. Of note, despite semantic memory gradually deteriorates with Alzheimer's disease pathological process, in the current task, relatively preserved performance can be supported by relative preservation of the most important regions for semantic memory, namely in superior lateral temporal lobe in early stage of Alzheimer's disease (Buckner et al., 2005; Dalla Barba et al., 1998) and the relatively automatic retrieval promoted by forced-choice recognition in our task.

\subsection{Subjective judgments}

\subsubsection{Subjective judgments for hits}

The proportions of episodic and semantic hits preceded by each subjective judgement in each group are shown in Figure 3.

Episodic task. A within-group paired-sample Student T-test revealed that, in AD patients, Prediction_low_hits was significantly higher than Prediction_high_hits $(P<.05)$. Betweengroups Student T-test revealed that Prediction_low_hits was significantly higher in AD patients than in HO $(P<.05)$. Other prediction-recognition variables were not significantly greater in $\mathrm{AD}$ patients than in $\mathrm{HO}$.

Semantic task. A within-group paired-sample Student T-test revealed that, in AD patients, the difference between Prediction_low_hits and Prediction_high_hits was not significant $(P<$ .05). Furthermore, between-groups Student T-test revealed that Prediction_low_hits and Prediction_high_hits did not differ from the respective same proportions (Prediction_low_hits and Prediction_high_hits) in HO.

[Figure 3 about here]

Genon et al. 
Thus, our behavioral results suggest that the pattern of subjective judgment before objective successful recognition is characterized by pessimistic prediction of failure (i.e. higher proportion of Prediction_low_hits) in AD patients.

\subsection{Correlation between judgments for hits and age and dementia}

Pearson correlation analyses showed that, in $\mathrm{AD}$ patients, predictive judgments for episodic hits (Prediction_low_hits and Prediction_high_hits) did not correlate with age, education and total score at the Mattis Dementia Rating Scale. Similarly, in these patients, judgments for semantic hits did not correlate with age and total score at the Mattis Dementia Rating Scale. In contrast, Pearson correlation analyses revealed that, in $\mathrm{HO}$ participants, predictive judgments for episodic hits (Prediction_low_hits and Prediction_high_hits) correlated with age, as shown by a significant negative correlation between Prediction_high_hits and age [r $=$ $-.55 ; P<.05]$ and a marginally significant positive correlation between Prediction_low_hits and age $[\mathrm{r}=.489 ; P=.05]$. Other correlations in $\mathrm{HO}$ participants were not significant.

\subsection{Correlation between episodic memory measures and grey matter density in AD}

\subsubsection{Multivariate analysis (regular behavioural PLS)}

We first explored the pattern of gray matter networks that covaried with the set of episodic memory measures of interest (Prediction_low_hits, Prediction_high_hits, Hits). As illustrated in Figure 4, only one latent variable showed a significant contribution to the covariance (explained crossblock covariance $75.04 \%, P=0.030$ ). This LV showed high positive correlation with Prediction_low_hits $(\mathrm{r}=0.83)$ and negative correlation with Prediction_high_hits $(r=-0.73)$, but null correlation with hits $(r=-0.04)$. We therefore assumed that this LV captures the relationship between GMV and the pessimistic pattern of prediction in $\mathrm{AD}$ patients. The brain regions where this pattern was most reliably identified included the right medial temporal lobe (with both hippocampus and parahippocampal 
cortex), posterior cingulate cortex and ventrolateral prefrontal cortex. These results are reported in Table 1 and illustrated in Figure 4.

[Table 1 and Figure 4 about here]

\subsubsection{Univariate analysis (SPM GLM analysis)}

Our behavioural analysis revealed that the difference in prediction patterns in AD patients and HO was significant when considering Prediction_low_hits and our exploratory cognitivemorphologic analysis suggested that this variable was highly related to the significant LV in PLS model, we then focused on this variable in the SPM GLM univariate cognitivemorphologic analysis. Accordingly, only Prediction_low_hits showed significant negative relationship with GMV in three neighbouring clusters (TFCE FWE corrected $P<.05$ ) located in the right ventrolateral prefrontal cortex (VLPFC), at the junction of inferior and middle frontal gyri (see Table 1). Importantly, the location of those clusters overlapped with the wider cluster revealed by the multivariate analysis as illustrated in Figure 5a providing evidence that the VLPFC cluster identified in PLS analysis was driven by Prediction_low_hits. Visualisation of adjusted correlation plots between GMV at the clusters' peak and Prediction_low_hits suggested that the relationship was not driven by outliers/extreme values as illustrated in Figure $5 \mathrm{~b}$.

[Figure 5 about here]

\section{$\underline{\text { 3.5. Additional cognitive-morphologic correlations in an independent healthy population }}$}

Our GLM analysis did not reveal any significant correlation between Prediction_low_hits/Prediction_high_hits and grey matter volume in HOfmri. This null 
result in healthy older people suggests that the significant correlations found in our AD sample reflect a brain-behavior relationship pattern that is specific to AD patients.

\section{Discussion}

In an ecologic task formed by recently presented unfamous people (episodic items) and famous people (semantic items) sharing similar demographic characteristics, we observed specific metacognitive judgments' pattern for episodic items in AD patients when compared to HO. While in the semantic task, AD patients showed a similar profile of judgements than HO participants, in the episodic task, they showed greater proportions of pessimistic predictions when considering successful retrieval (i.e. Prediction_low_hits). The multivariate analysis (behavioural PLS) using GM volume and behavioural performance revealed that the pattern of judgments before successful retrieval in $\mathrm{AD}$ patients was related to a mainly right lateralized pattern of GMV decrease in prefrontal and medial temporal regions. GLM further confirmed the significant negative relationship between GMV in right ventrolateral prefrontal cortex (VLPFC) and Prediction_low_hits.

According to Koriat, several processes underlie a predictive judgment at retrieval stage, such as in the traditional FOK paradigm (Koriat, 1993; Koriat and Levy-Sadot, 2001). First, a quick and preliminary FOK is driven by the cue's familiarity or fluency (Paynter et al., 2009). In the present study, this first FOK is performed based on the familiarity/fluency of the presented face. According to the instruction given to the participant, we assume that the latter would choose Null_feeling if he/she hasn't got any memory of the face. When the cue (i.e., the face in the present study) induces a FOK, memory search is initiated. This latter process, then, results in accumulation of partial information, such as contextual details. The quantity 
and accessibility of associated information retrieved is in turn assessed by monitoring processes to determine the FOK and infer about recognition performance (Thomas et al., 2011). In the present study, we assume that efficient monitoring of the amount of partial information (such as "the names contains several "i", "the name sounds like an object name") and/or accessibility of the memory trace will lead to hits being accompanied by Prediction_high. In contrast, altered monitoring of the amount of partial information or accessibility of the memory trace will result in hits being accompanied by Prediction_low. The pattern of successful name recognition with pessimistic prediction judgments (Prediction_low_hits) for the person's name in AD patients suggests that the monitoring process that allows insight and inferences about one's own online episodic memory functioning is impaired in those patients.

Of note, metacognitive predictive judgments on one's performance rely on several processes that may be considered beyond the traditional FOK paradigm and theoretical conceptualization. In the FOK paradigm, the participant assesses his/her own "memory feeling" based on his/her online insight in his/her own memory. In a predictive judgment on one's own subsequent memory performance, an additional process of inference/decision making should be engaged to predict one's performance based on one's FOK. That is, we can also consider one additional neurocognitive mechanism that has not been considered in previous model based on traditional FOK paradigm: inferences making process. Accordingly, we hypothesize that Prediction_low for successful retrieval (Prediction_low_hits) may be driven by dysfunctional inference making processes in AD patients.

In a related view, a pattern of prediction of poor recognition performance (pattern of prediction_low) may be fed by a general low confidence and/or conservative judgments attitude for one's episodic memory's abilities rather than a direct output of poor feeling-of-

Genon et al. 
knowing. According to this view, the higher pattern of Prediction_low_hits in AD patients would reflect general low confidence of AD patients in their memory abilities expressed as pessimistic judgments. In other words, according to this view, the observed pattern would be influenced by general beliefs of memory retrieval failure in those patients. Such beliefs might have been promoted by personal exposure to $\mathrm{AD}$ patients, perceived $\mathrm{AD}$ thread and negative stereotype more pronounced in the environment of our AD patients (Kinzer and Suhr, 2016; Suhr and Kinkela, 2007). Nevertheless, several studies show that approximately half of the AD patients are not aware of their behavioral deficits (e.g. Clare et al., 2010; Mograbi et al., 2012). Furthermore, in the current study, the pattern of underconfidence in AD patients is specific to episodic items while AD patients show a profile similar to the one of $\mathrm{HO}$ in the semantic task with minor proportion of pessimistic predictions (less than $25 \%$ at the group level). That is, the unawareness of memory difficulties generally observed in the AD population and the profile of specific pessimistic judgments on memory performance for episodic items do not support the hypothesis of a general trend of $\mathrm{AD}$ patients to underestimate themselves or to adapt conservative strategies in their judgments. In contrast, the hypothesis of inaccurate beliefs of failure specific to episodic memory tasks in AD patients cannot be rejected. Despite this hypothesis needs further empirical support to be considered alone as accounting for our results, we consider that some specific beliefs can interfere with metacognitive monitoring in our task.

In addition to considering the influence of specific beliefs in the pattern of pessimistic prediction of episodic memory failure in our AD patients, we consider that this pattern might be an epiphenomenon related to our experimental setting. Indeed, by capitalizing on efficient and deep encoding based on iterative cued immediate recall of the target name, we involuntary expose our patients to their memory recall failures for name materials. That is, we consider the hypothesis that our encoding procedure has triggered transient awareness of Genon et al. 
memory failure for name recall in AD patients. Previous studies have suggested that, despite $\mathrm{AD}$ are not aware of their memory impairment, they can adapt their metacognitive judgments according to their very recent experience (within a few minutes delay, not beyond) with the memory task (Duke et al., 2002; Mimura and Yano, 2006; Schmitter-Edgecombe and Seelye, 2011; Stewart et al., 2010). Importantly, previous studies that have assessed online metacognitive predictions of recognition performance in patients with Mild Cognitive Impairment (MCI) and $\mathrm{AD}$ patients have used a traditional FOK paradigm, in which the participants experience recall failure before indicating their FOK judgments. Nevertheless, those studies have suggested that MCI and $\mathrm{AD}$ patients tend to overestimate their memory abilities in their FOK (Perrotin et al., 2007; Souchay et al., 2002). Such inconsistencies across studies raise the question of the extent to which online metacognitive judgments are influenced by task features (as already pointed out in the framework of memory postdictions in AD patients by (Dodson et al., 2011)). In the current study, this issue could be assessed by examining the within-subject relationship between encoding variables and metacognitive prediction at recognition, but encoding variables (such as the number of iterations and encoding time) have not been recorded. Therefore, the issue of the influence of the task features on metacognitive judgments in $\mathrm{AD}$ patients is still an open question that should be investigated in future studies. For the time being, we would mainly consider the (more parsimonious) hypothesis of impaired online monitoring processes of the memory content in AD patients to account for the observed pattern of pessimistic predictions in AD patients.

Little is known about the neurocognitive monitoring mechanisms accompanying retrieval of recently formed episodic memories in AD patients. In a previous study (Genon et al., 2013), we found that AD patients showed activation of the PCC (which is assumed to be a crucial cortical hub in higher cognitive processes such as episodic memory retrieval (Leech and Sharp, 2014)) during episodic retrieval as healthy older participants did, while they showed 
impaired functional connectivity between PCC on the one hand and hippocampus and lateral

prefrontal cortex (LPFC) on the other hand. This suggested that impaired controlled/conscious retrieval of recent episodic memories in $\mathrm{AD}$ patients might be related to disconnection within a wide brain network. Furthermore, a recent study showed that impaired general awareness of one's own functioning in $\mathrm{AD}$ was related not only to functional changes within cortical midline structures involved in self-referential processes (such as the PCC), but also to disconnection between these regions and the medial temporal lobe (Perrotin et al., 2015). In line with this idea, in the present study, PLS analyses revealed that the altered pattern of judgments for successful retrieval in AD patients is associated with GMV decrease in a wide brain network including the medial temporal lobe, the PCC and the VLPFC. Further analyses using a univariate analysis whose significance was assessed with permutations confirmed the significant association between VLPFC GMV and pessimistic predictions in AD patients, suggesting that this region may play an important role in their metacognitive dysfunction.

Previous studies have revealed that patients with frontal lesions showed inaccurate metacognitive predictive judgments on episodic memories suggesting that some damage to frontal regions alter metacognitive monitoring (Pannu and Kaszniak, 2005). In the present study, higher pattern of inadequate predictive judgment is related to reduced grey matter volume in the VLPFC. Importantly, previous fMRI studies in healthy participants have shown that activation in this region was modulated by the degree of FOK in an episodic memory task, that is, greater FOK was related to higher activation, during an episodic memory task ((Maril et al., 2003), MNI coordinates: 45, 30, 6; (Chua et al., 2009) MNI coordinates: 45, 39, 3)). Importantly, activation in this region has also been found to be modulated by the degree of FOK in a semantic task ((Kikyo et al., 2002), MNI coordinates: 45, 42, 18)). One can therefore assume that activation in this region during FOK is not related to the engagement of episodic memory retrieval processes but rather reflects the engagement of high cognitive 
processes common to both episodic and semantic metacognitive predictive judgments. In this framework, this region has been found to be activated in a wide range of higher cognitive processes such as reasoning ((Kroger et al., 2002), MNI coordinates: 4623 32), decision making ((Labudda et al., 2008), MNI coordinates: 5032 24) and memory retrieval monitoring ((Cabeza et al., 2002), MNI coordinates: 4419 31; (Cabeza et al., 2003), MNI coordinates: 44 24 24: (Kensinger and Schacter, 2005), MNI coordinates: 3936 17; (Nee and Jonides, 2009), MNI coordinates: 5026 26). This region has been therefore assumed to support monitoring of the retrieval process with verification and checking operations (Cabeza et al., 2003; Mitchell and Johnson, 2009). Thus, the right VLPFC is engaged in monitoring processes accompanying retrieval, such as metacognitive inferences elaboration. The present study showed that pessimistic pattern of failure prediction is related to decreased grey matter volume in this region supporting monitoring processes for inferences elaboration in AD.

In conclusion, for the first time, our study revealed that $\mathrm{AD}$ patients show a pattern of pessimistic, impaired prediction of failure before subsequent successful memory retrieval that is specific to episodic memories and related to GM decrease in the right VLPFC. We hypothesized that such profile is related to impaired monitoring of episodic memory retrieval conceptualized as altered insight on available information or altered subsequent inference process on memory performance supported by right VLPFC. Future studies should better characterize the monitoring dysfunction resulting in inaccurate metacognitive judgments during episodic memory retrieval in $\mathrm{AD}$ patients.

\section{$\underline{\text { Acknowledgments }}$}

This work was supported by grants from the Belgian Foundation for Research on Alzheimer's disease (SAO-FRMA, grant \#08612); the King Baudouin Foundation; the Belgian National Funds for Scientific Research (FNRS); Belgian Inter-University Attraction Poles P7/11, a

Genon et al. 
French Speaking Community Concerted Research Action (ARC) and the Fonds Leon Fredericq of the University of Liege.

\section{Author Contributions:}

SG, MJ, SC, FC, CB and ES contributed to the conception and design of the study. SG, JS, MJ, CB and ES contributed to acquisition of the data. SG, JS, FC, MB and ES contributed to the analysis of data.

\section{$\underline{\text { Potential Conflicts of Interest }}$}

All authors reported no biomedical financial interests or potential conflicts of interest.

\section{References}

Amunts, K., Kedo, O., Kindler, M., Pieperhoff, P., Mohlberg, H., Shah, N., Habel, U., Schneider, F., Zilles, K. Cytoarchitectonic mapping of the human amygdala, hippocampal region and entorhinal cortex: intersubject variability and probability maps. Anatomy and embryology 210 (5-6): 343-352, 2005.

Amunts, K., Schleicher, A., Bürgel, U., Mohlberg, H., Uylings, H., Zilles, K. Broca's region revisited: cytoarchitecture and intersubject variability. Journal of Comparative Neurology 412 (2): 319-341, 1999.

Backman, L., Lipinska, B. Monitoring of general knowledge: evidence for preservation in early Alzheimer's disease. Neuropsychologia 31 (4): 335-345, 1993.

Bludau, S., Eickhoff, S.B., Mohlberg, H., Caspers, S., Laird, A.R., Fox, P.T., Schleicher, A., Zilles, K., Amunts, K. Cytoarchitecture, probability maps and functions of the human frontal pole. NeuroImage 93 Pt 2: 260-275, 2014.

Buckner, R.L., Snyder, A.Z., Shannon, B.J., LaRossa, G., Sachs, R., Fotenos, A.F., Sheline, Y.I., Klunk, W.E., Mathis, C.A., Morris, J.C., Mintun, M.A. Molecular, structural, and functional characterization of Alzheimer's disease: Evidence for a relationship between default activity, amyloid, and memory. The Journal of Neuroscience 25 (34): 7709-7717, 2005.

Burton, A.M., White, D., McNeill, A. The Glasgow face matching test. Behav Res Methods 42 (1): 286-291, 2010.

Cabeza, R., Anderson, N.D., Locantore, J.K., McIntosh, A.R. Aging gracefully: compensatory brain activity in high-performing older adults. Neuroimage 17 (3): 1394-1402, 2002.

Cabeza, R., Locantore, J.K., Anderson, N.D. Lateralization of prefrontal activity during episodic memory retrieval: evidence for the production-monitoring hypothesis. Journal of cognitive neuroscience 15 (2): 249-259, 2003. 
Chua, E.F., Schacter, D.L., Sperling, R.A. Neural correlates of metamemory: a comparison of

Clare, L., Whitaker, C.J., Nelis, S.M. Appraisal of memory functioning and memory performance in healthy ageing and early-stage Alzheimer's disease. Neuropsychology, development, and cognition. Section B, Aging, neuropsychology and cognition 17 (4): 462491, 2010.

Dalla Barba, G. Recognition memory and recollective experience in Alzheimer's disease. Memory 5 (6): 657-672, 1997.

Dalla Barba, G., Parlato, V., Jobert, A., Samson, Y., Pappata, S. Cortical Networks Implicated in Semantic and Episodic Memory: Common or Unique? Cortex; a journal devoted to the study of the nervous system and behavior 34 (4): 547-561, 1998.

Deichmann, R., Schwarzbauer, C., Turner, R. Optimisation of the 3D MDEFT sequence for anatomical brain imaging: technical implications at 1.5 and 3 T. Neuroimage 21: 757-767, 2004.

Diedrichsen, J., Balsters, J.H., Flavell, J., Cussans, E., Ramnani, N. A probabilistic MR atlas of the human cerebellum. Neuroimage 46 (1): 39-46, 2009.

Dodson, C.S., Spaniol, M., O'Connor, M.K., Deason, R.G., Ally, B.A., Budson, A.E. Alzheimer's disease and memory-monitoring impairment: Alzheimer's patients show a monitoring deficit that is greater than their accuracy deficit. Neuropsychologia 49 (9): 26092618, 2011.

Duke, L.M., Seltzer, B., Seltzer, J.E., Vasterling, J.J. Cognitive components of deficit awareness in Alzheimer's disease. Neuropsychology 16 (3): 359, 2002.

Eakin, D.K., Hertzog, C., Harris, W. Age invariance in semantic and episodic metamemory: Both younger and older adults provide accurate feeling-of-knowing for names of faces. Aging, Neuropsychology, and Cognition 21 (1): 27-51, 2014.

Gallo, D.A., Cramer, S.J., Wong, J.T., Bennett, D.A. Alzheimer's disease can spare local metacognition despite global anosognosia: revisiting the confidence-accuracy relationship in episodic memory. Neuropsychologia 50 (9): 2356-2364, 2012.

Genon, S., Bahri, M.A., Collette, F., Angel, L., d'Argembeau, A., Clarys, D., Kalenzaga, S., Salmon, E., Bastin, C. Cognitive and neuroimaging evidence of impaired interaction between self and memory in Alzheimer's disease. Cortex; a journal devoted to the study of the nervous system and behavior 51: 11-24, 2014.

Genon, S., Collette, F., Feyers, D., Phillips, C., Salmon, E., Bastin, C. Item familiarity and controlled associative retrieval in Alzheimer's disease: an fMRI study. Cortex; a journal devoted to the study of the nervous system and behavior 49 (6): 1566-1584, 2013.

Kanai, R., Rees, G. The structural basis of inter-individual differences in human behaviour and cognition. Nature Reviews Neuroscience 12 (4): 231-242, 2011.

Kensinger, E.A., Schacter, D.L. Retrieving accurate and distorted memories: neuroimaging evidence for effects of emotion. Neuroimage 27 (1): 167-177, 2005.

Kikyo, H., Ohki, K., Miyashita, Y. Neural correlates for feeling-of-knowing: an fMRI parametric analysis. Neuron 36 (1): 177-186, 2002.

Kinzer, A., Suhr, J.A. Dementia worry and its relationship to dementia exposure, psychological factors, and subjective memory concerns. Applied neuropsychology. Adult 23 (3): 196-204, 2016.

Koriat, A. How do we know that we know? The accessibility model of the feeling of knowing. Psychol. Rev. 100: 609-639, 1993.

Koriat, A., Levy-Sadot, R. The combined contributions of the cue-familiarity and accessibility heuristics to feelings of knowing. Journal of experimental psychology. Learning, memory, and cognition 27 (1): 34-53, 2001. 
Kroger, J.K., Sabb, F.W., Fales, C.L., Bookheimer, S.Y., Cohen, M.S., Holyoak, K.J. Recruitment of anterior dorsolateral prefrontal cortex in human reasoning: a parametric study of relational complexity. Cerebral cortex 12 (5): 477-485, 2002.

Labudda, K., Woermann, F.G., Mertens, M., Pohlmann-Eden, B., Markowitsch, H.J., Brand, M. Neural correlates of decision making with explicit information about probabilities and incentives in elderly healthy subjects. Exp Brain Res 187 (4): 641-650, 2008.

Leech, R., Sharp, D.J. The role of the posterior cingulate cortex in cognition and disease. Brain : a journal of neurology 137 (1): 12-32, 2014.

Lipinska, B., Backman, L. Feeling-of-knowing in fact retrieval: further evidence for preservation in early Alzheimer's disease. Journal of the International Neuropsychological Society : JINS 2 (4): 350-358, 1996.

Maril, A., Simons, J.S., Mitchell, J.P., Schwartz, B.L., Schacter, D.L. Feeling-of-knowing in episodic memory: an event-related fMRI study. NeuroImage 18 (4): 827-836, 2003.

Mattis, S. Dementia Rating Scale. NFER-Nelson: Windsor, England, 1973.

McIntosh, A.R., Lobaugh, N.J. Partial least squares analysis of neuroimaging data: applications and advances. NeuroImage 23 Suppl 1: S250-263, 2004.

McKhann, G., Drachman, D., Folstein, M., Katzman, R., Price, D., Stadlan, E.M. Clinical diagnosis of Alzheimer's disease: report of the NINCDS-ADRDA Work Group under the auspices of Department of Health and Human Services Task Force on Alzheimer's Disease. Neurology 34: 939-944, 1984.

McKhann, G.M., Knopman, D.S., Chertkow, H., Hyman, B.T., Jack, C.R., Kawas, C.H., Klunk, W.E., Koroshetz, W.J., Manly, J.J., Mayeux, R. The diagnosis of dementia due to Alzheimer's disease: Recommendations from the National Institute on Aging-Alzheimer's Association workgroups on diagnostic guidelines for Alzheimer's disease. Alzheimer's and Dementia 7 (3): 263-269, 2011.

Mimura, M., Yano, M. Memory impairment and awareness of memory deficits in early-stage Alzheimer's disease. Reviews in the neurosciences 17 (1-2): 253-266, 2006.

Mitchell, K.J., Johnson, M.K. Source monitoring 15 years later: what have we learned from fMRI about the neural mechanisms of source memory? Psychological bulletin 135 (4): 638, 2009.

Mograbi, D.C., Ferri, C.P., Sosa, A.L., Stewart, R., Laks, J., Brown, R., Morris, R.G. Unawareness of memory impairment in dementia: a population-based study. International psychogeriatrics / IPA 24 (6): 931-939, 2012.

Moulin, C.J.A., James, N., Perfect, T.J., Jones, R.W. Knowing what you cannot recognise: Further evidence for intact metacognition in Alzheimer's disease. Aging, Neuropsychology, and Cognition 10 (1): 74-82, 2003.

Nee, D.E., Jonides, J. Common and distinct neural correlates of perceptual and memorial selection. Neuroimage 45 (3): 963-975, 2009.

Nelson, T.O., Narens, L. Metamemory: A theoretical framework and some new findings. in: Bower, G.H. (Ed.), The Psychology of Learning and Motivation. Academic Press: San Diego, 1990. pp. 125-173.

Pannu, J.K., Kaszniak, A.W. Metamemory experiments in neurological populations: A review. Neuropsychol.Rev. 15 (3): 105-130, 2005.

Paynter, C.A., Reder, L.M., Kieffaber, P.D. Knowing we know before we know: ERP correlates of initial feeling-of-knowing. Neuropsychologia 47 (3): 796-803, 2009.

Perrotin, A., Belleville, S., Isingrini, M. Metamemory monitoring in mild cognitive impairment: Evidence of a less accurate episodic feeling-of-knowing. Neuropsychologia 45: 2811-2826, 2007.

Perrotin, A., Desgranges, B., Landeau, B., Mezenge, F., La Joie, R., Egret, S., Pelerin, A., de la Sayette, V., Eustache, F., Chetelat, G. Anosognosia in Alzheimer disease: Disconnection between memory and self-related brain networks. Ann Neurol 78 (3): 477-486, 2015. 
Schmitter-Edgecombe, M., Seelye, A.M. Predictions of verbal episodic memory in persons with Alzheimer's disease. Journal of clinical and experimental neuropsychology 33 (2): 218225, 2011.

Schnyer, D.M., Nicholls, L., Verfaellie, M. The role of VMPC in metamemorial judgments of content retrievability. J. Cogn. Neurosci. 17 (5): 832-846, 2005.

Smith, S.M., Nichols, T.E. Threshold-free cluster enhancement: addressing problems of smoothing, threshold dependence and localisation in cluster inference. Neuroimage 44 (1): 83-98, 2009.

Souchay, C., Isingrini, M., Gil, M. Alzheimer's disease and feeling-of-knowing in episodic memory. Neuropsychologia 40: 2386-2396, 2002.

Souchay, C., Isingrini, M., Gil, R. Metamemory monitoring and Parkinson's disease. Journal of clinical and experimental neuropsychology 28 (4): 618-630, 2006.

Souchay, C., Moulin, C.J. Memory and consciousness in Alzheimer's disease. Current Alzheimer research 6 (3): 186-195, 2009.

Stewart, G., McGeown, W.J., Shanks, M.F., Venneri, A. Anosognosia for memory impairment in Alzheimer's disease. Acta Neuropsychiatrica 22 (4): 180-187, 2010.

Suhr, J.A., Kinkela, J.H. Perceived threat of Alzheimer disease (AD): the role of personal experience with AD. Alzheimer disease and associated disorders 21 (3): 225-231, 2007.

Thomas, A.K., Bulevich, J.B., Dubois, S.J. Context affects feeling-of-knowing accuracy in younger and older adults. Journal of Experimental Psychology: Learning, Memory, and Cognition 37 (1): 96, 2011.

Walsh, N.D., Dalgleish, T., Lombardo, M.V., Dunn, V.J., Van Harmelen, A.-L., Ban, M., Goodyer, I.M. General and specific effects of early-life psychosocial adversities on adolescent grey matter volume. NeuroImage: Clinical 4: 308-318, 2014.

Wegelin, J.A. A Survey 0fPartial Least Squares (PLS) Methods, with Emphasis on the TwoBlock Case. 2000.

Ziegler, G., Dahnke, R., Winkler, A.D., Gaser, C. Partial least squares correlation of multivariate cognitive abilities and local brain structure in children and adolescents. NeuroImage 82: 284-294, 2013. 
Figure 1. Experimental procedure for the episodic items. Prediction-recognition phase was similar for the semantic items (not illustrated here). The episodic item represented here was used as an example during the administration of the instructions. The face's photography comes from the Glasgow Unfamiliar Face Database (GUFD, (Burton et al., 2010)).

Figure 2. Memory performance expressed as mean percentages of hits in experimental groups. Standard errors are represented by error bars. *Performance of AD patients is significantly lower than performance of $\mathrm{HO}$ at $\mathrm{P}<.05$.

Figure 3. Proportion of hits preceded by each level of predictions in each experimental group *Proportions in $\mathrm{AD}$ patients is significantly lower than respective proportion in $\mathrm{HO}$ at $\mathrm{P}<.05$.

Figure 4. Multivariate PLS results of relationships between behavioral variables on regional GMV. Right bottom plot illustrates the percentage of explained covariance for each latent variable (LV). Only LV1, whose brain pattern is illustrated here, showed significance over random permutations with probability of the permuted values being greater than observed over 600 permutations $<.05$.

Figure 5. A. Rendering of significant regions showing a relationship between grey matter volume and pattern of judgments in the multivariate analysis (yellow) and the significant regions showing a negative relationship between Prediction_low_hits and GMV in the univariate analysis (red). B. Scatter plot of grey matter volume in the significant right prefrontal ventrolateral cluster (y) and Prediction_low_hits (x) after ajusting for age and dementia ratings. 
Table 1. Brain regions in which grey matter volume shows significant negative covariance with behavioral measures.

\begin{tabular}{|c|c|c|c|c|c|c|}
\hline Region & Side & $\begin{array}{c}\text { Overlap with } \\
\text { cytoarchitectonic area }\end{array}$ & $\mathrm{x}$ & $\mathrm{y}$ & $\mathrm{z}$ & $\begin{array}{l}\text { Cluster } \\
\text { size }\end{array}$ \\
\hline \multicolumn{7}{|l|}{ PLS analysis } \\
\hline Inferior/middle frontal & $\mathrm{R}$ & Area $45^{\text {(Amunts et al., 1999) }}$ & 45 & 27 & 13 & 3790 \\
\hline Middle orbital gyrus & $\mathrm{L}$ & $\mathrm{Fp} 1^{\text {(Bludau et al., 2014) }}$ & -27 & 45 & 11 & 547 \\
\hline Posterior frontal & $\mathrm{R}$ & NA & 27 & -9 & 55 & 399 \\
\hline /precentral gyrus & $\mathrm{L}$ & NA & -41 & 2 & 39 & 914 \\
\hline $\mathrm{PCC}$ & M & NA & & & & \\
\hline Inferior temporal gyrus & $\mathrm{R}$ & NA & 52 & -54 & -14 & 301 \\
\hline Medial temporal lobe & $\mathrm{R}$ & Hippocampus ${ }^{\text {(Amunts et al., 2005) }}$ & 31 & -20 & -22 & 1724 \\
\hline \multirow[t]{3}{*}{ Cerebellum } & $\mathrm{L}$ & $\begin{array}{l}\text { Lobule VIIa,b/VIIIa, }{ }^{\text {(Diedrichsen et }} \\
\text { al., 2009) }\end{array}$ & -33 & -61 & -44 & 3001 \\
\hline & $\mathrm{R}$ & Lobule VIIa, $b^{\text {(Diedrichsen et al., 2009) }}$ & 34 & -75 & -44 & 688 \\
\hline & $\mathrm{R}$ & $\begin{array}{l}\text { Lobule VIIIa,b/IX/X } \mathrm{X}^{\text {(Diedrichsen et }} \\
\text { al., 2009) }\end{array}$ & 24 & -50 & -49 & 904 \\
\hline \multicolumn{7}{|l|}{ GLM analysis } \\
\hline Inferior frontal gyrus/ & $\mathrm{R}$ & Area $45^{\text {(Amunts et al., 1999) }}$ & 46 & 28 & 14 & 77 \\
\hline \multirow[t]{2}{*}{ Middle frontal gyrus } & $\mathrm{R}$ & NA & 46 & 37 & 16 & 26 \\
\hline & $\mathrm{R}$ & NA & 46 & 24 & 31 & 16 \\
\hline
\end{tabular}

Note. NA, not assigned; PCC, posterior cingulate cortex; PLS, partial least square; GLM, general linear model. PLS results are significant at $\mathrm{P}<.001$ (bootstrap threshold \pm 3.3 ) and GLM results are significant at $\mathrm{P}<.05$ corrected (Threshold-Free Cluster Enhancement). 


\section{Familiarisation phase}

\section{Man or woman?}

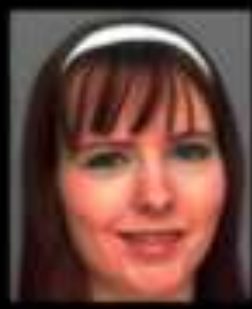

\section{Encoding phase}

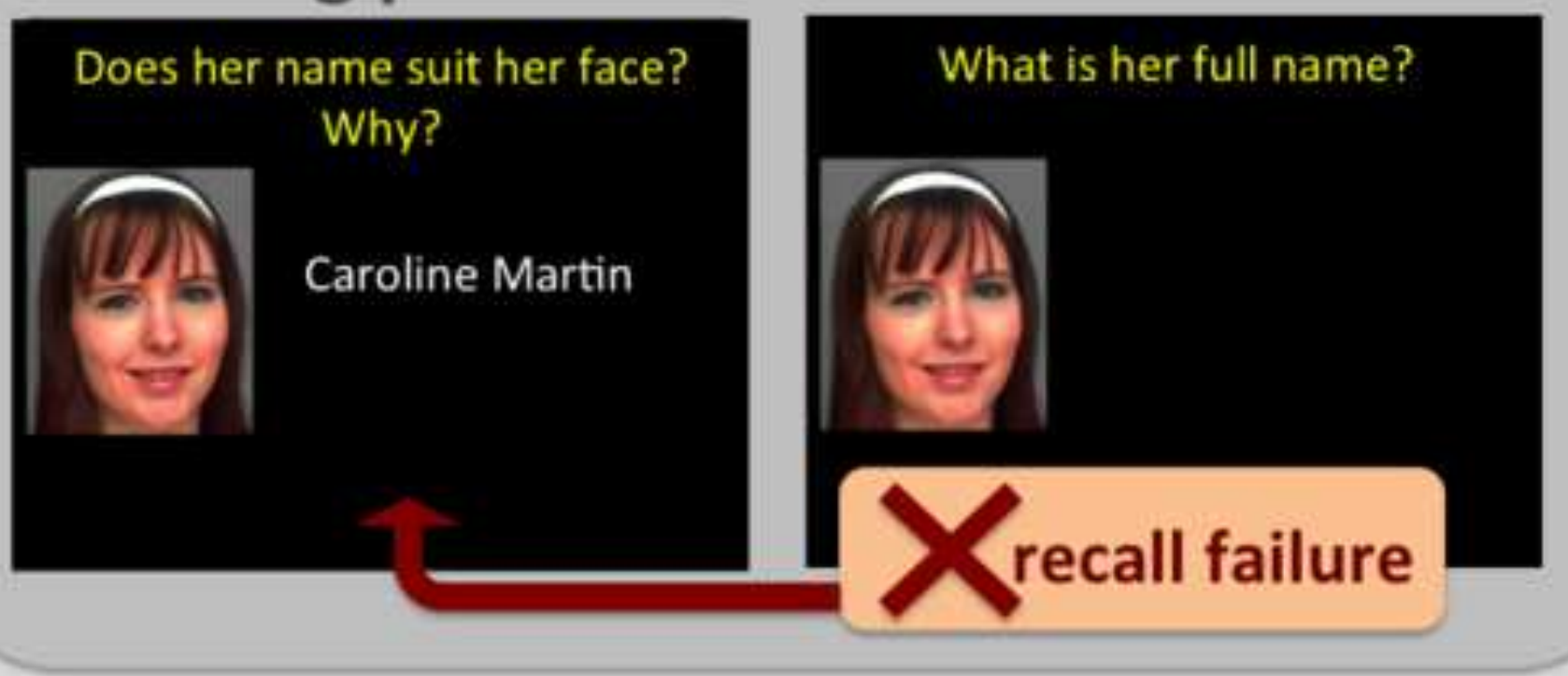

\section{Prediction-recognition phase}

Could you recognize her full name?

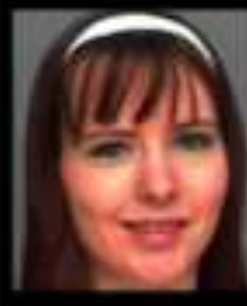

1. Not at all

2. Low probability

3. High probability

4. Yes, I know it
What is her name?

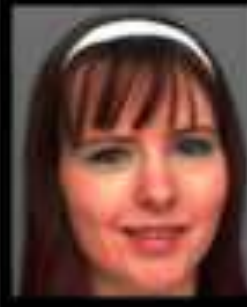

1. Caroline Ravin

2. Stéphanie Dupont

3. Stéphanie Rufon

4. Caroline Martin 
Episodic Semantic

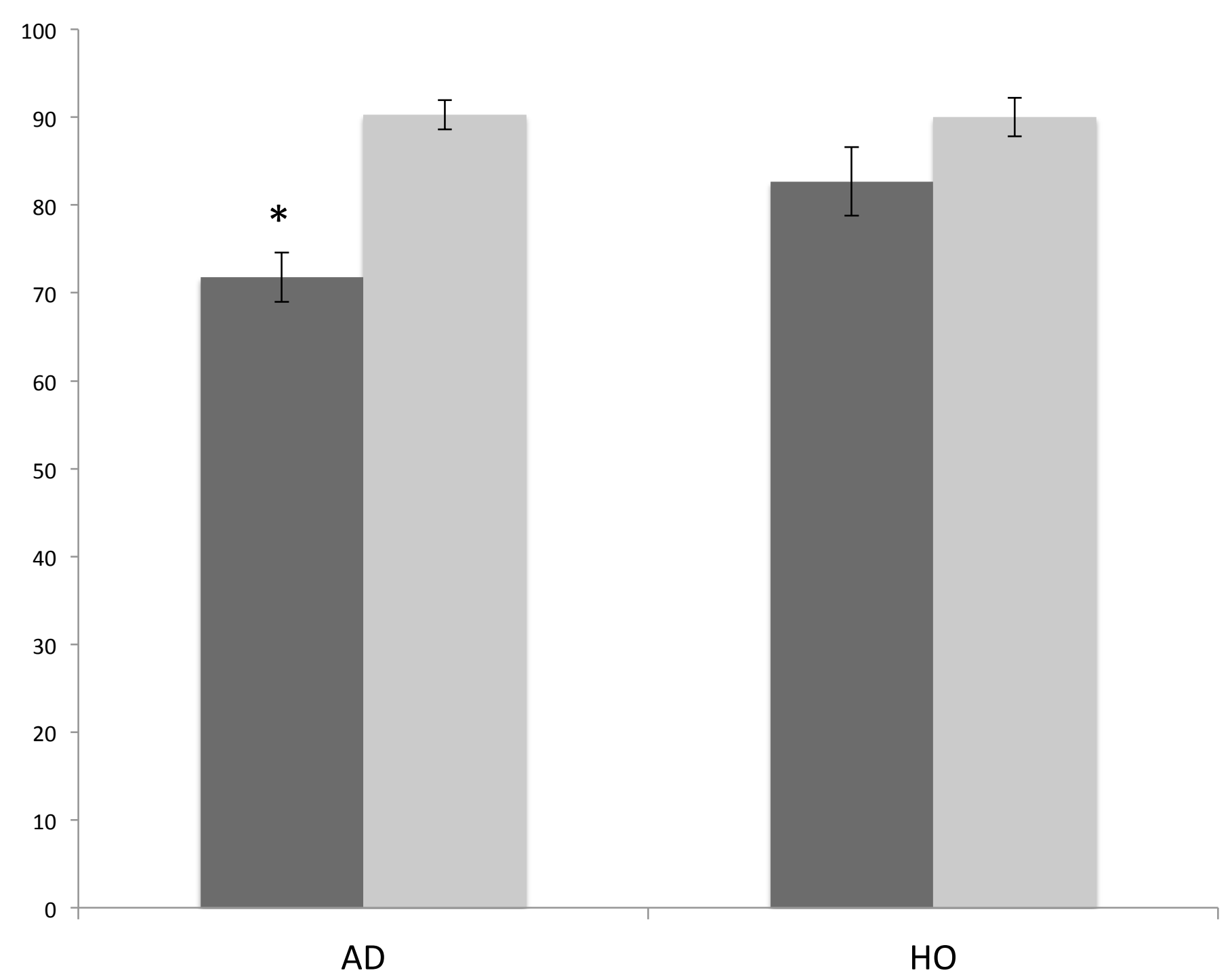


Figure 3
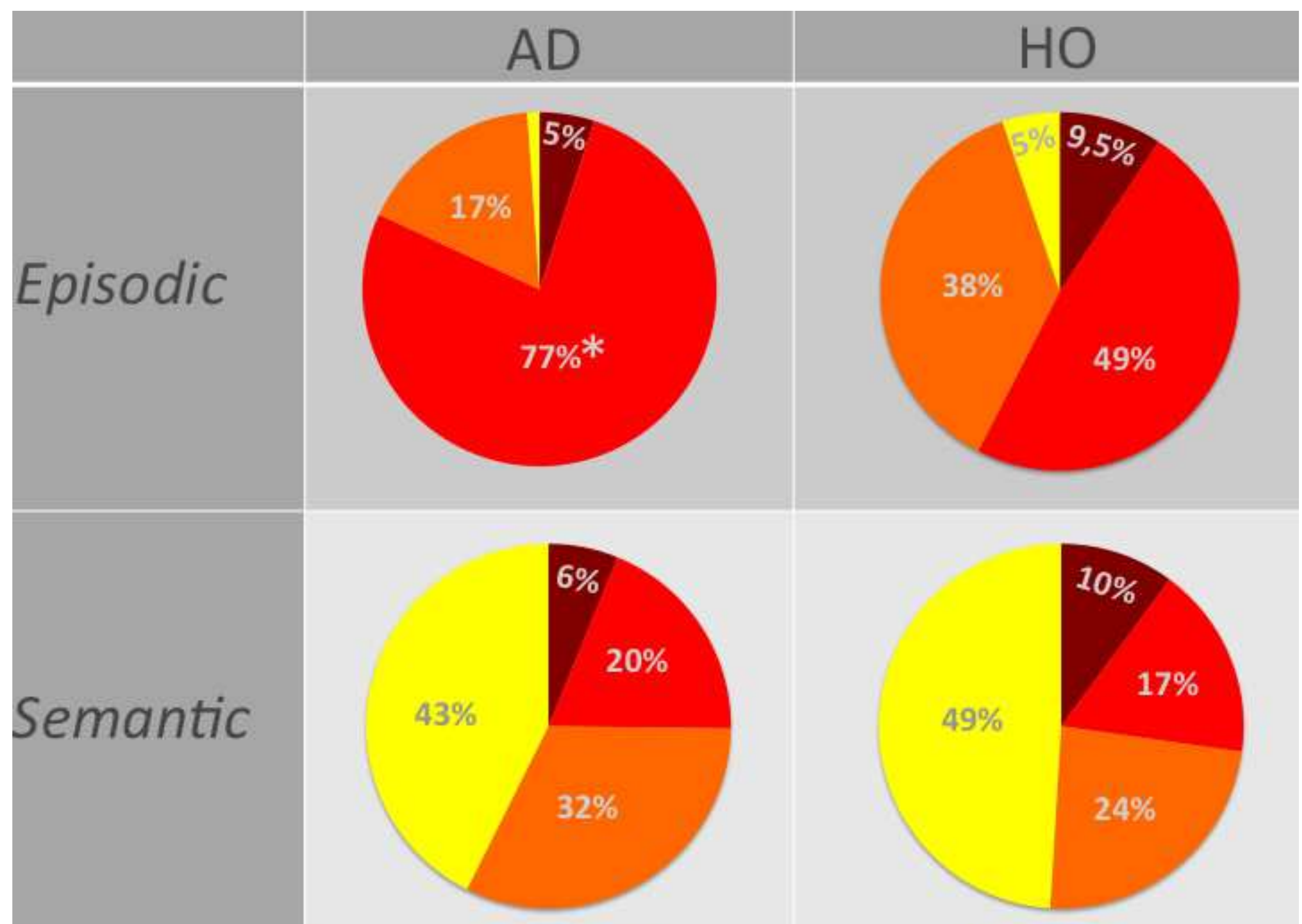

Null_feeling_hits

- Prediction_low_hits = Prediction_high_hits

Subjective_recall_hits 


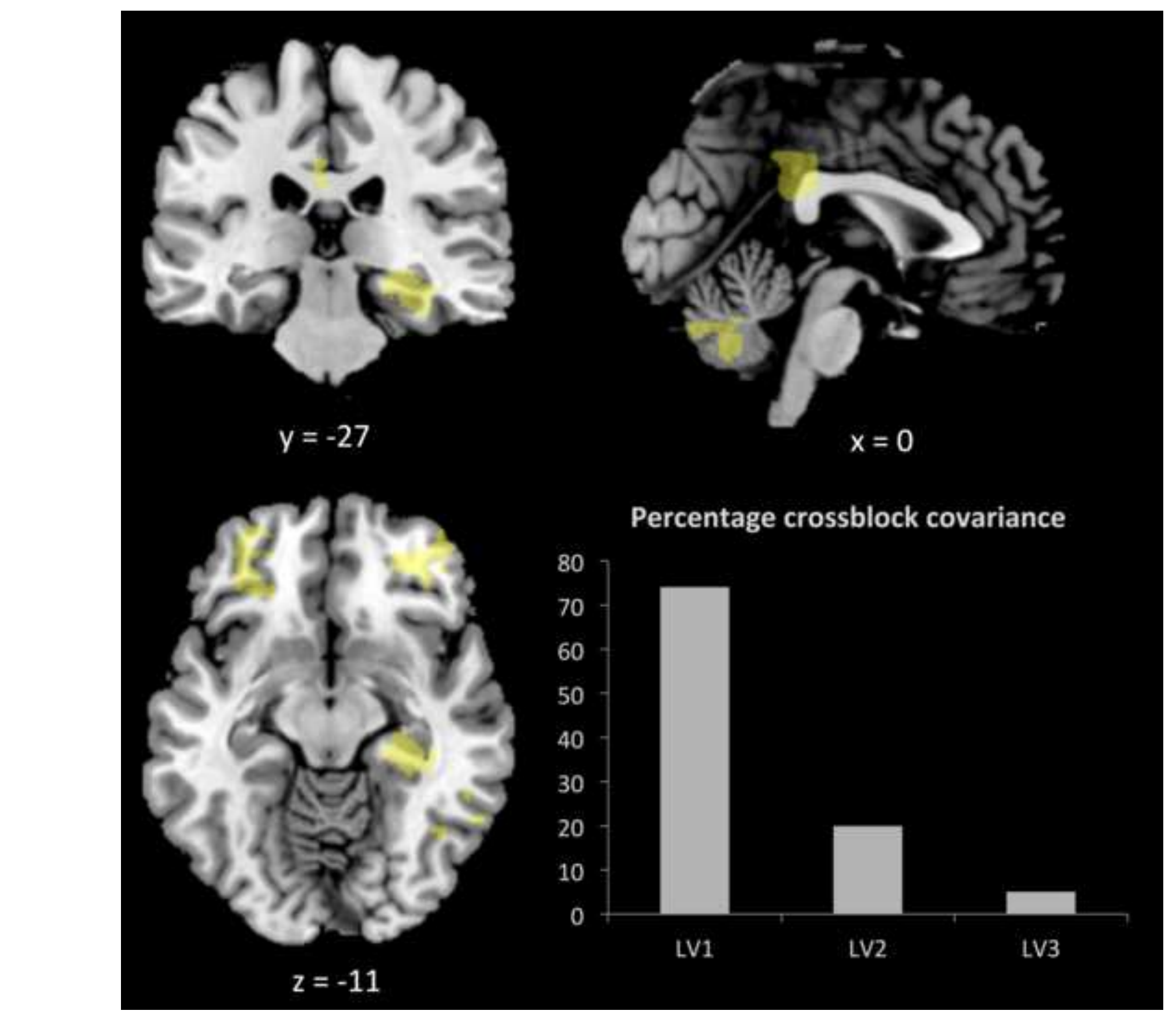


A

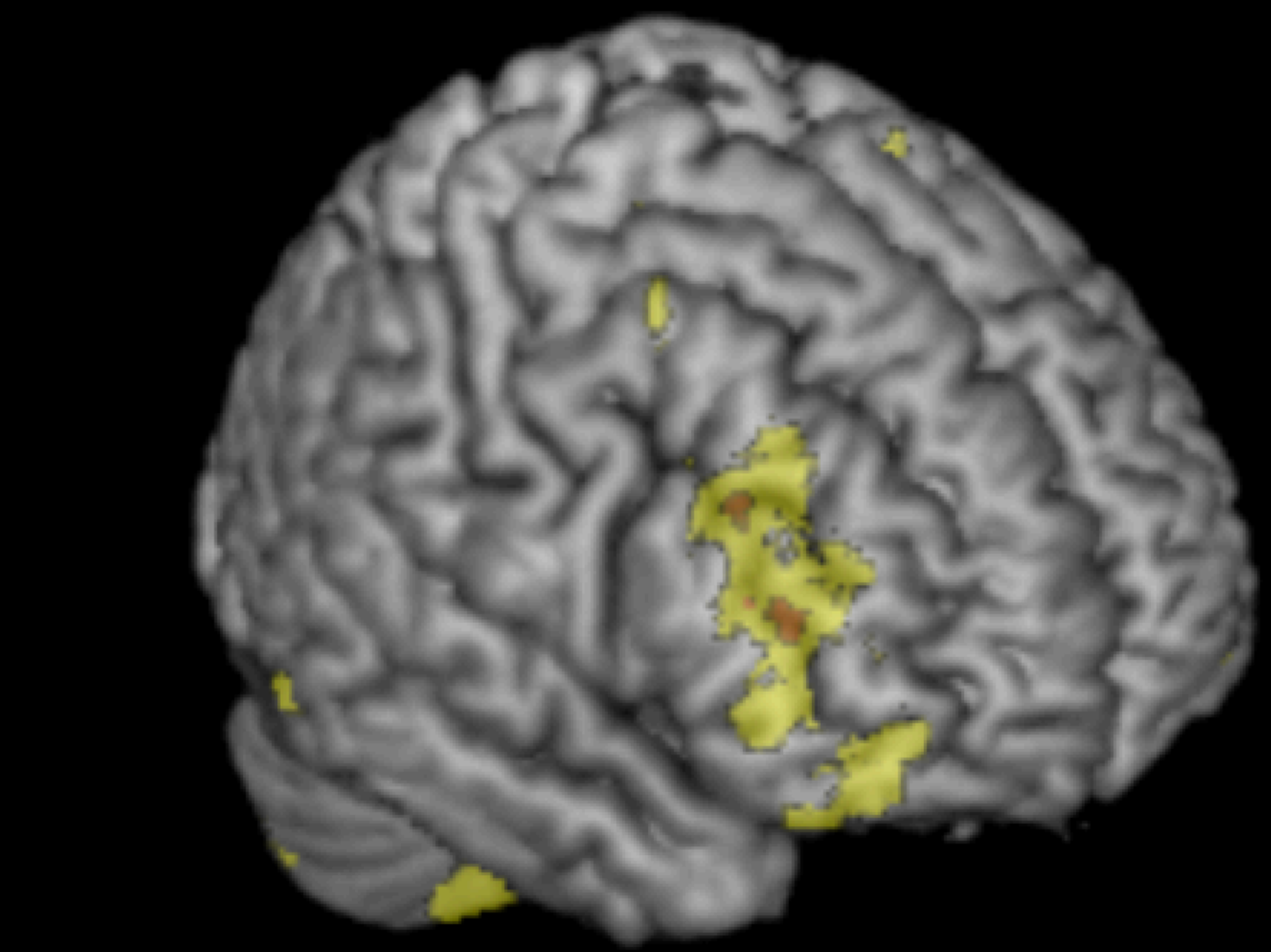

B

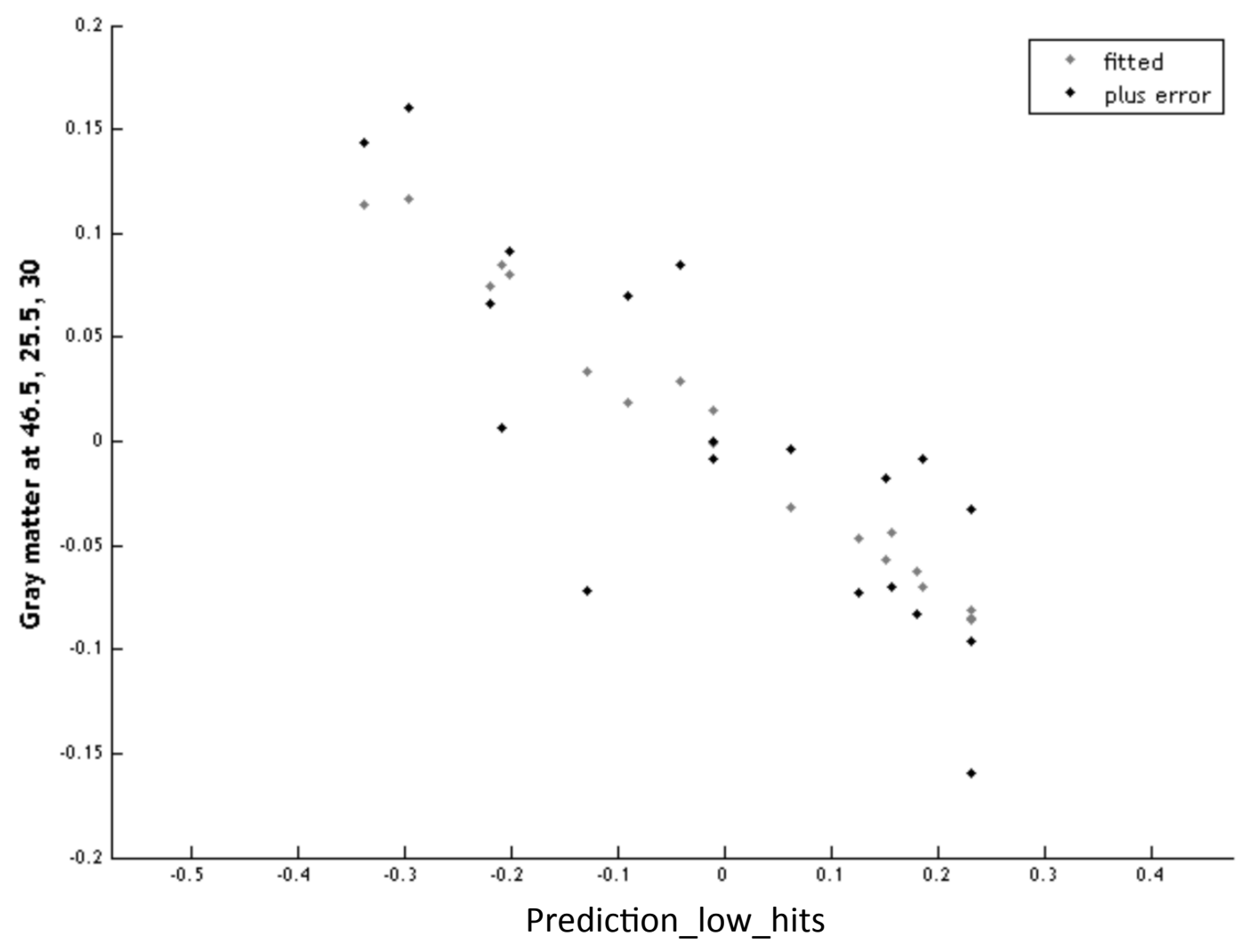


$\underline{\text { Relating pessimistic memory predictions to Alzheimer's disease brain structure }}$

Sarah Genon ${ }^{1,2}$, Jessica Simon ${ }^{1}$, Mohamed Ali Bahri ${ }^{1}$, Fabienne Collette ${ }^{1,6}$, Céline Souchay ${ }^{5}$, Mathieu Jaspar ${ }^{1}$, Christine Bastin ${ }^{1 *}$ and Eric Salmon ${ }^{1,4^{*}}$.

${ }^{1}$ GIGA-CRC in vivo imaging, University of Liège, Belgium

${ }^{2}$ Institute of Neuroscience und Medicine (INM-1), Research Centre Juelich, Juelich, Germany

${ }^{4}$ Memory Centre, Service of Neurology, CHU Liège, Belgium

${ }^{5}$ Laboratoire de Psychologie et Neurocognition CNRS UMR 5105, Université Pierre MendèsFrance, Grenoble, France

${ }^{6}$ Psychology and Cognitive Neuroscience research unit, University of Liège, Belgium

* Equally contributed

Running title: Metacognitive predictions in Alzheimer's disease

\section{Corresponding author:}

Sarah Genon, GIGA-CRC in vivo imaging, University of Liège, Quartier Agora, Allée du 6 Août, B30, 4000 Liège, Belgium, Telephone: 3243665475, Fax: 3243662946, Email: sarah.genon@ulg.ac.be. 
*Highlights

Highlights:

- AD patients showed pessimistic predictive judgments of their memory performance - This pattern is related to grey matter decrease in ventrolateral prefrontal cortex

- Impaired inferences on own functioning may be related to VLPFC perturbations in AD 